\title{
Magnetic Resonance Imaging in Giant Perianal Epidermoid
}

\author{
Aruna Raman Patil ${ }^{1} \quad$ Amit Bansal ${ }^{2} \quad$ Nilotpal Chakma ${ }^{3} \quad$ Ravishankar Bhat $^{3} \quad$ Swarna Shivakumar ${ }^{4}$ \\ ${ }^{1}$ Department of Radiology, Apollo Hospitals, Bengaluru, Karnataka, \\ India \\ ${ }^{2}$ Department of Surgical Gastroenterology, Apollo Hospitals, \\ Bengaluru, Karnataka, India \\ ${ }^{3}$ Department of Gastrosurgery, Apollo Hospitals, Bengaluru, \\ Karnataka, India \\ ${ }^{4}$ Department of Pathology, Apollo Hospitals, Bengaluru, Karnataka \\ India \\ Address for correspondence Aruna Raman Patil, MD, FRCR \\ Department of Radiology, Apollo Hospitals, Bannerghatta Road, \\ Bengaluru 560076, Karnataka, India \\ (e-mail: dr.arunarpatil@gmail.com).
}

\author{
Abstract \\ Keywords \\ - epidermoid \\ - perianal \\ - magnetic resonance \\ imaging \\ - presacral \\ - cyst \\ - developmental
}

Perianal epidermoid cyst is a rare entity and is commonly diagnosed late owing to its slow-growing nature. Imaging plays a vital role in diagnosis and differentiation from other cysts such as dermoid, tailgut, and rectal duplication cysts. Magnetic resonance imaging (MRI) is the preferred modality as uncomplicated cases show typical signal changes. Diffusion-weighted imaging has a definite additional role. We report a case of a giant perianal epidermoid diagnosed on MRI and successfully managed surgically.

\section{Introduction}

Perianal epidermoid is a rare developmental cyst and is commonly diagnosed late due to its slow-growing nature. It is a benign condition with an extremely rare chance of malignant transformation. Differentiation from other perianal or retrorectal cysts such as dermoid, tailgut and duplication cysts is important. Among the various gamuts of imaging, magnetic resonance imaging (MRI) offers better characterization and details on extension and is the preferred modality for presurgical workup. Apart from typical signal changes in an uncomplicated epidermoid, diffusion-weighted imaging (DWI) in MRI has a definite additional role.

\section{Case Report}

A 70-year-old male presented with a painless enlarging mass in the right perineal region for 3 years. There was no other significant complaint except for occasional episodes of incomplete bowel emptying. There was no history of recent fever or back pain. No history of previous trauma was elicited. Physical examination revealed a firm, slightly mobile mass in the right perianal region with normal overlying skin. No induration or discharging sinus was seen. On per rectal examination, a firm mass was felt in the right lateral anal wall with overlying normal mucosa. Proctoscopic examination was within normal limits. MRI (MR Ingenia Elition 3.0T, Philips Healthcare, Koninklijke Philips N.V) with intravenous contrast (Omniscan-Gadodiamide $0.1 \mathrm{mmol} / \mathrm{kg}$, intravenous injection; GE Healthcare AS, Oslo, Norway) was done to evaluate the extent and mass characteristics.

MRI revealed a well-defined smoothly encapsulated oblong-shaped presacral cystic mass with a large right lateral component paralleling the anal canal and rectum. The mass measured $15 \mathrm{~cm}$ in craniocaudal length and $7 \mathrm{~cm}$ in width. The mass extended to the ischioanal and ischiorectal

\section{published online}

August 5, 2021
DOI https://doi.org/

$10.1055 / \mathrm{s}-0041-1731966$ ISSN 2581-9933 (c) 2021. Indian Society of Gastrointestinal and Abdominal Radiology. This is an open access article published by Thieme under the terms of the Creative Commons Attribution-NonDerivative-NonCommercial-License, permitting copying and reproduction so long as the original work is given appropriate credit. Contents may not be used for commercial purposes, or adapted, remixed, transformed or built upon. (https://creativecommons.org/licenses/by-nc-nd/4.0/). Thieme Medical and Scientific Publishers Pvt. Ltd. A-12, 2nd Floor, Sector 2, Noida-201301 UP, India 
fossa on the right side up to the skin caudally. The anal canal and rectum were compressed by the lesion, displaced to left with splaying and thinning of the external sphincter and urogenital diaphragm. The mass was isointense on T1-weighted images and hyperintense on T2-weighted images with hypointense "flake" like scattered contents ( - Fig. 1A-D). No enhancing solid component was seen. No communication to the vertebra or visceral or cutaneous communication was

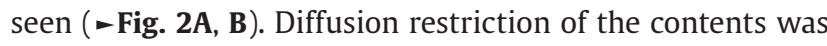
seen on DWI sequences ( - Fig. 3A, B). On contrast administration, smooth peripheral wall enhancement was noted ( - Fig. 3C). The imaging features of a cystic lesion with contents showing diffusion restriction along with nonenhancing hypointense flakes were classical of an epidermoid cyst. In view of the large size and compressive effects, excision of the cyst was done under general anesthesia with a perianal incision ( - Fig. 4A). The cyst was resected with the capsule in toto of the subcutaneous tissue, internal and external sphincter. The mass was outside the mesorectal fascia ( - Fig. 4B, C). Cut open cyst revealed cheesy material and keratin flakes ( - Fig. 4D). Histopathological examination showed the cyst to be lined by keratinizing stratified squamous epithelium with granular layer and sheets of foreign body giant cells at places ( - Fig. 5A, B). The lumen was filled with lamellated keratin material. The histopathology was consistent with an epidermoid.

\section{Discussion}

Peri anorectal or retrorectal cysts in adults are a rare entity and are commonly developmental. Developmental cysts include epidermoid, dermoid, and enteric cysts. ${ }^{1}$ Epidermoid cysts are slowly growing unilocular benign lesions lined by stratified squamous epithelium and are a result of misplaced ectodermal tissues during embryogenesis. The contents are as a result a mixture of desquamated epithelium

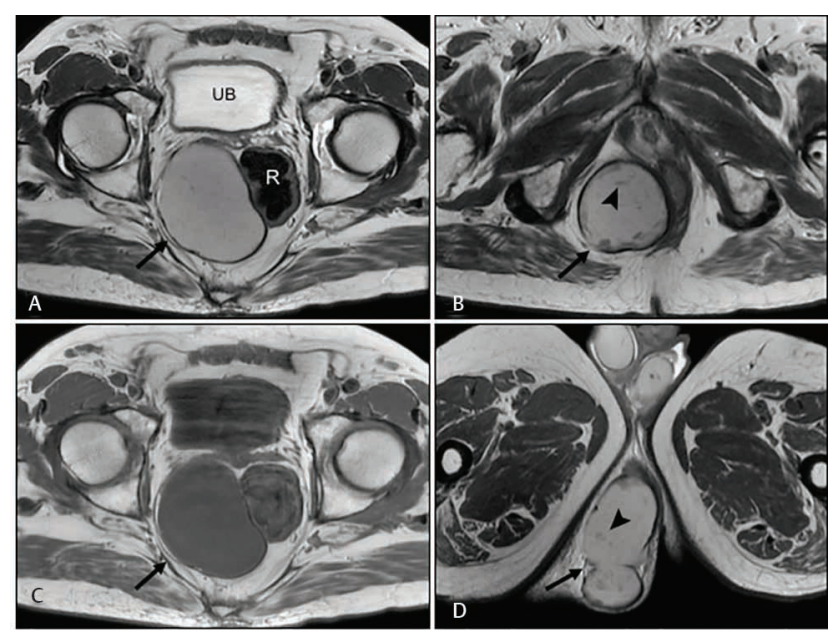

Fig. 1 Axial T2-weighted images at the level of hip joints (A), ischial tuberosity (B), and perineum (D) show a large well encapsulated hyperintense mass (black arrow) along the right lateral aspect of the rectum and anal canal with isointense debris like contents (arrowheads). (C) Axial T1-weighted image at the same level as (A) shows the mass to be isointense. $R$, rectum; UB, urinary rectum.
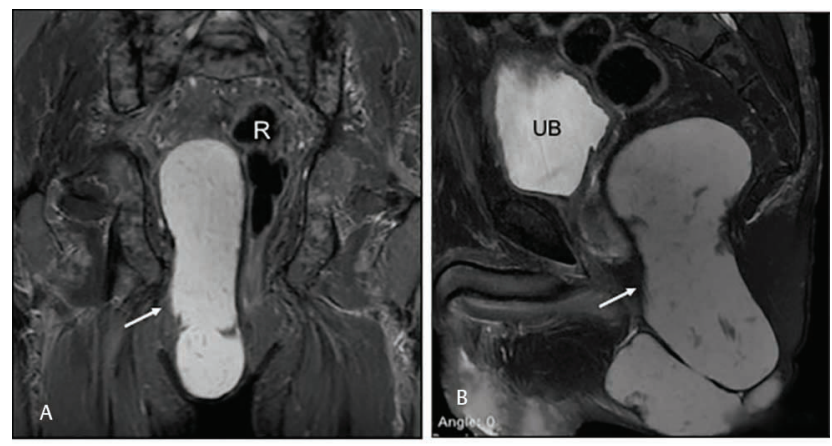

Fig. 2 (A) Coronal spin-echo short inversion time inversion-recovery image shows lesion to be hyperintense and oblong in shape along the right anorectal region extending into the perineum (arrow). (B) Sagittal T2 image with fat saturation shows the lobulated nature and extension of the lesion along with internal contents. No communication with vertebra is seen. $R$, rectum; UB, urinary rectum.

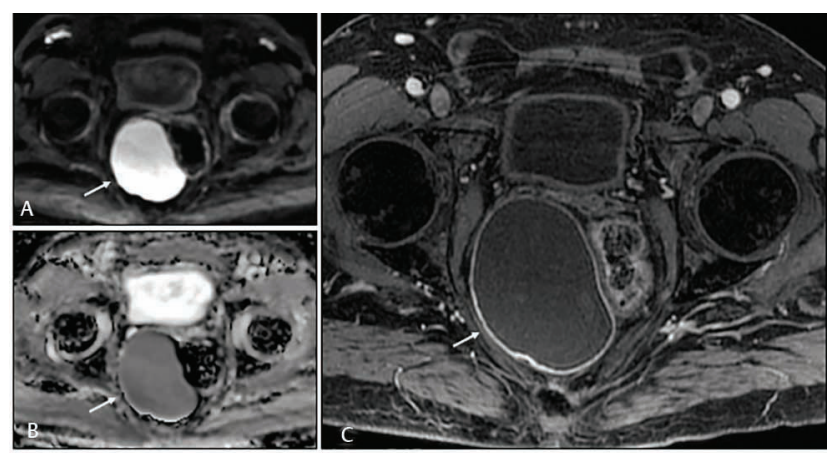

Fig. 3 (A, B) Diffusion-weighted imaging (DWI) (b=800) and apparent diffusion coefficient images show diffusion restriction of the contents (arrow). (C) Axial T1 postgadolinium contrast with fat saturation shows smooth thin peripheral enhancement of the cyst wall (arrow).

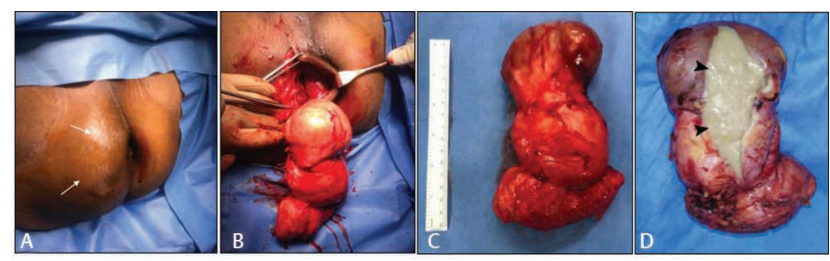

Fig. 4 (A) Right perianal swelling (arrows). (B) Open perineal resection of the mass. (C). Completely resected well-encapsulated mass measuring $\sim 15 \mathrm{~cm}$ long. (D) Cut open mass revealing cheesy material suggesting epidermoid (arrowheads).

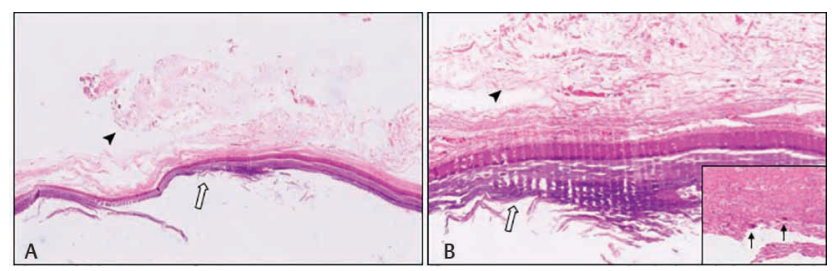

Fig. 5 (A) Hematoxylin and eosin stain, 4X scanner view and (B) hematoxylin and eosin stain, 10X low power view show a cyst wall lined by keratinizing stratified squamous epithelium (white arrow) with granular layer and the lumen is filled with lamellated keratin material (arrowhead). Inset shows sheets of foreign body giant cells at places (black arrows). 
and keratin ${ }^{2}$ that have characteristics imaging features. Many of them are asymptomatic and when large cause mass effect on rectum, ureter, or urinary bladder. ${ }^{3}$ Other uncommon presentations include infection, chronic discharging sinus, perianal fistula, and bleeding ${ }^{3}$

Malignant transformation of presacral cysts is rare and commonly results in adenocarcinoma. Primary cystic presacral squamous cell carcinoma or squamous cell carcinoma arising from a presacral epidermoid cyst has been previously described only in two reports in the literature. ${ }^{4}$

Imaging plays a vital role in the diagnosis of this condition. Ultrasonography features include uniloculated smooth-walled mass with heterogeneous contents. No solid components or internal vascularity are seen. Computed tomography establishes precise location with the lesion showing isodense or hyperdense contents with smooth wall and enhancement. Generally, lesional fat and calcification is absent and if seen it favors dermoid cyst. ${ }^{5}$ MRI is the imaging modality of choice. Location of the lesion, relation to adjacent structures, and internal characteristics are better assessed on MRI. Epidermoid cysts are isointense on T1-weighted sequences and hyperintense on T2-weighted sequences with internal hypointense foci representing keratin flakes. The latter feature is characteristic for epidermoid and useful to differentiate from other mimics like dermoid cyst or tailgut cyst. On contrast administration, thin peripheral enhancement is seen..$^{5-7}$ The use of DWI adds value in diagnosis as the contents show diffusion restriction. ${ }^{8}$

Presacral space is defined as that between the mesorectal fascia anteriorly and presacral fascia posteriorly and extends laterally up to the level ureter and iliac vessels. ${ }^{9}$ Presacral masses can be approached on the basis of whether the lesion shows sacral involvement, extends to sacral foramen, presents as a rectal mass per se, or occurs as an isolated presacral pathology. Differentiating the mass as solid or cystic can narrow down the differentials, as entirely cystic isolated presacral masses include epidermoid, dermoid, tailgut cyst, lymphatic malformation, and rectal duplication cyst. Dermoid has derivatives from skin appendages like tooth and is heterogenous with predominant fat and cystic areas. Tailgut cyst and lymphatic malformation are generally multiloculated with clear fluid contents with or without internal echoes and does not show diffusion restriction. ${ }^{10}$

The definitive treatment of a retrorectal epidermoid cyst is complete surgical excision along with the wall to prevent recurrence of rare malignant transformation. ${ }^{11,12}$ The surgical approach is either anterior abdominal or posterior (transsacral, transperineal) or combined according to the location and size of the mass. In our case, transperineal approach was used. Laparoscopic approach is practiced at some places. ${ }^{12}$

Perianal epidermoid is a rare entity and MRI is the preferred modality for diagnosis and should be used to map and characterize any retrorectal or peri anorectal lesions. Complete resection of the cyst is the treatment of choice.

\section{Conflict of Interest}

None declared.

\section{References}

1 Dahan H, Arrivé L, Wendum D, Docou le Pointe H, Djouhri H, Tubiana J-M. Retrorectal developmental cysts in adults: clinical and radiologic-histopathologic review, differential diagnosis, and treatment. Radiographics 2001;21(3):575-584

2 Yang DM, Yoon MH, Kim HS, et al. Presacral epidermoid cyst: imaging findings with histopathologic correlation. Abdom Imaging 2001;26(1):79-82

3 Jha A, Khalid M, Gupta P, Saud M, Gupta G. Presacral epidermoid cyst in an elderly female, mistaken for ovarian cystadenoma. J Obstet Gynaecol India 2014;64(Suppl 1):118-120

4 Gaud U, Goyal T, Shukla M, Kumar V, Pandey M. Synchronous occurrence of adenocarcinoma of the rectum with squamous cell carcinoma of a retrorectal cyst: report of a case and review of the literature. BMJ Case Rep 2009;2009. DOI: 10.1136/ bcr.12.2008.1398

5 Al- Shoura R, Malaekah H, Al Bassam W. Giant retrorectal epidermoid cyst masquerading as a perianal swelling. Case Rep Surg 2020;2020. DOI: 10.1155/2020/5750382

$6 \mathrm{Kim} \mathrm{H}, \mathrm{Kim} \mathrm{JH}$, Lim JS, et al. MRI findings of rectal submucosal tumors. Korean J Radiol 2011;12(4):487-498

7 Nicolay S, De Schepper A, Pouillon M. Epidermal inclusion cyst of the perianal region. JBR-BTR 2014;97(3):166-167

8 Turkay R, Caymaz I, Yildiz B, Livaoglu A, Turkey B, Bakir B. A rare case of epidermoid cyst of perineum: diffusion-weighted MRI and ultrasonography findings. Radiol Case Rep 2015;8(1):59310.2484/rcr.v8i1.593

9 Hain KS, Pickhardt PJ, Lubner MG, Menias CO, Bhalla S. Presacral masses: multimodality imaging of a multidisciplinary space. Radiographics 2013;33(4):1145-1167

10 Yang DM, Park CH, Jin W, et al. Tailgut cyst: MRI evaluation. AJR Am J Roentgenol 2005;184(5):1519-1523

11 Hosseini-Nik H, Hosseinzadeh K, Bhayana R, Jhaveri KS. MR imaging of the retrorectal-presacral tumors: an algorithmic approach. Abdom Imaging 2015;40(7):2630-2644

12 Duclos J, Maggiori L, Zappa M, Ferron M, Panis Y. Laparoscopic resection of retrorectal tumors: a feasibility study in 12 consecutive patients. Surg Endosc 2014;28(4):1223-1229 